CASE REPORT

\title{
Enhancing Retention Using Overdentures with Bar Attachment: A Case Report
}

\author{
Nishita Narendra Ranadive ${ }^{1}$, Jyoti B Nadgere ${ }^{2}$, Janani lyer ${ }^{3}$, Pinak J Rathi ${ }^{4}$
}

\begin{abstract}
Aim: The aim of this case report is to present a case with overdenture attachment.

Background: Loss of teeth in the mandibular arch leads to increased resorption of the alveolar bone resulting in instability of the mandibular ridge. The principles of preventive prosthodontics enable us to preserve the remaining natural teeth and use them as abutments for added retention, improved proprioception, and masticatory efficiency.

Case description: The patient was evaluated clinically and radiographically and a tentative jaw relation was recorded. The patient following extraction presented with maxillary completely edentulous arch and mandibular arch with 33,34,43, and 44. The abutment teeth were prepared to receive a bar attachment on 33 and 43 and dome-shaped preparation to receive metal copings on 34 and 44 . Final impressions were made using zinc oxide eugenol paste and addition silicone impression material for the maxillary and the mandibular arch, respectively. Following the fabrication of the attachments, the jaw relation was recorded. Teeth arrangement was assessed and dentures were processed.

Conclusion: The patient was successfully rehabilitated with a maxillary conventional complete denture and an overdenture attachment Hader bar with 33 and 43 to improve the stability of the mandibular denture.

Clinical significance: This technique of overdenture attachment with the Hader bar allowed splinting of the abutment teeth along with stress
\end{abstract} distribution subsequently benefiting the patient psychologically.

Keywords: Attachments, Hader bar, Overdenture.

Journal of Contemporary Dentistry (2019): 10.5005/jp-journals-10031-1250

\section{BACKGROUND}

In dentistry, it is an imperial task for a dentist to fabricate a stable and retentive mandibular denture. Many patients who present themselves as potent complete denture patients have few retained teeth in their mouth. DeVan's golden statement: "Perpetual preservation of what remains is more important than the meticulous replacement of what is missing" is still true. ${ }^{1}$ Applying the principles of preventive prosthodontics, these teeth can be saved from extraction and can be used as abutments for "overdenture prosthesis." ${ }^{2,3}$ Overdentures are defined as "Any removable dental prosthesis that covers and rests on one or more remaining natural teeth, the roots of natural teeth, and/or dental implants" given by GPT $9 .^{4}$

The prime objective behind overdenture prosthesis is to preserve the remaining natural teeth, alveolar bone, proprioception, and the attached gingiva supporting the denture.,

Retention in overdentures can be increased by adding attachments which can be extraradicular or intraradicular. ${ }^{7}$ Extraradicular attachments can be physically classified into studs and bar attachments. ${ }^{8}$

This case report describes a tooth-supported overdenture with the Hader bar fabricated for a mandibular ridge.

\section{Case Description}

A 60-year-old male patient reported at the Department of Prosthodontics with a chief complaint of multiple missing teeth, inability to chew, and unaesthetic look.

The patient gave a history of angioplasty and was under aspirin medication since 2 years. Past dental history revealed that the patient had undergone extraction of his upper posterior teeth 6-7 years back and upper anterior teeth 2 months back.
${ }^{1-4}$ Department of Prosthodontics, Mahatma Gandhi Mission's Dental College and Hospital, Crown and Bridge, Navi Mumbai, Maharashtra, India Corresponding Author: Nishita Narendra Ranadive, Department of Prosthodontics, Mahatma Gandhi Mission's Dental College and Hospital, Crown and Bridge, Navi Mumbai, Maharashtra, India, Phone: +91 7506269615, e-mail: nishitaranadive@gmail.com

How to cite this article: Ranadive NN, Nadgere JB, et al. Enhancing Retention using Overdentures with Bar Attachment: A Case Report. J Contemp Dent 2019;9(1):37-43.

Source of support: Nil

Conflict of interest: None

Extraoral examination revealed an ovoid facial form and a convex facial profile without loss of vertical. Clinical examination revealed that the teeth present were $13,32,33,34,43$, and 44 . A grossly decayed 13 presenting with grade II mobility was observed. Grade II mobility with 32 was seen. A square-shaped mandibular arch with wellrounded ridges was noted (Fig. 1). An orthopantomogram (OPG) along with the intraoral periapical (IOPA) radiographs were used to assess the endodontic and periodontal prognosis of the teeth.

Oral prophylaxis was carried out and it was planned to extract 13 and 32 due to poor periodontal prognosis. Prior to the extraction of these teeth, a diagnostic irreversible hydrocolloid impression was made and a tentative jaw relation was recorded at the vertical maintained by the remaining natural teeth and mounted on a semiadjustable Hanau articulator with a facebow (Fig. 2). Diagnostic teeth arrangement was done. Interarch space available was assessed to be $41 \mathrm{~mm}$ with class I ridge relationship.

The various treatment options presented to the patient were the following:

() The Author(s). 2019 Open Access This article is distributed under the terms of the Creative Commons Attribution 4.0 International License (https://creativecommons. org/licenses/by-nc/4.0/), which permits unrestricted use, distribution, and non-commercial reproduction in any medium, provided you give appropriate credit to the original author(s) and the source, provide a link to the Creative Commons license, and indicate if changes were made. The Creative Commons Public Domain Dedication waiver (http://creativecommons.org/publicdomain/zero/1.0/) applies to the data made available in this article, unless otherwise stated. 

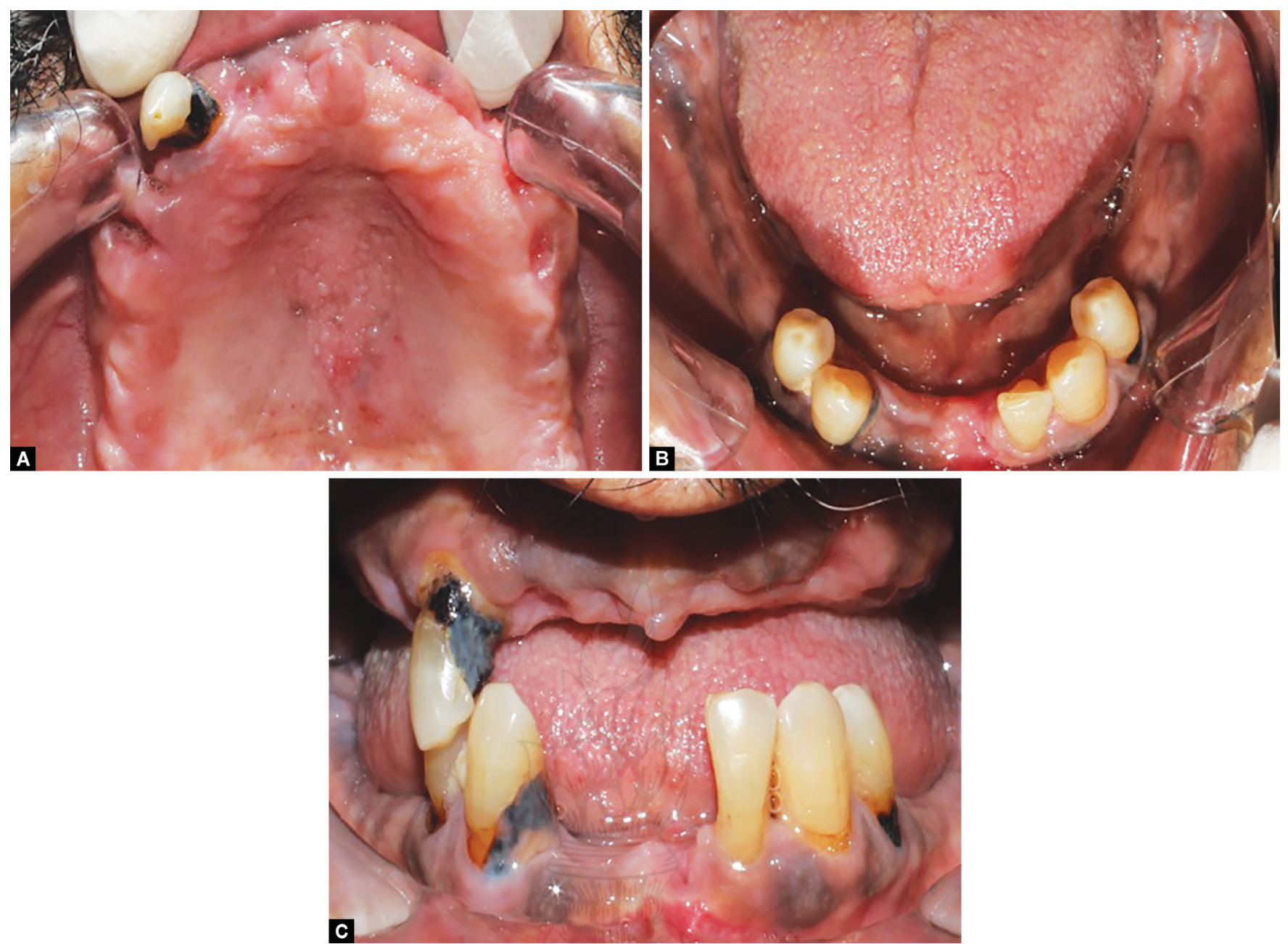

Figs $1 \mathrm{~A}$ to $\mathrm{C}$ : Preoperative intraoral

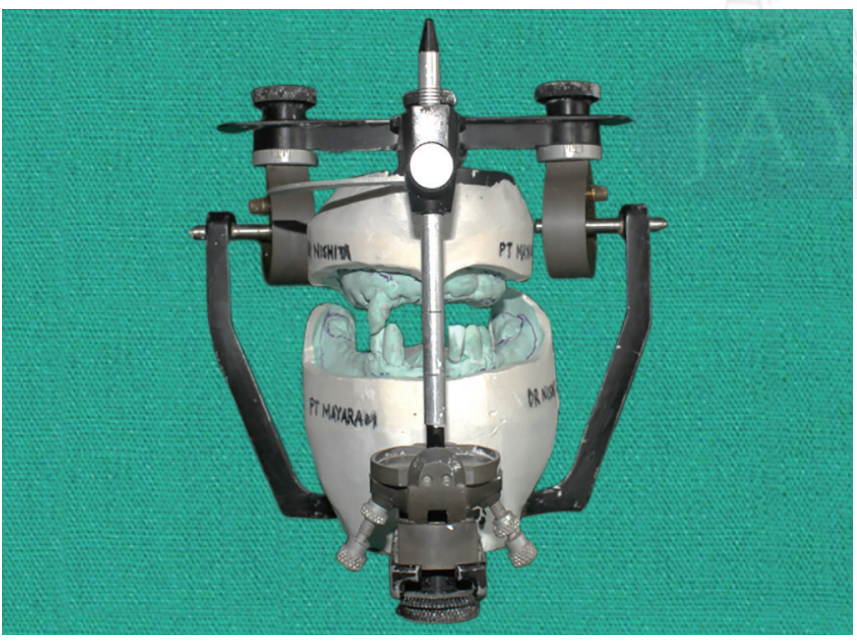

Fig. 2: Diagnostic mounting

Maxillary arch

- Conventional complete denture

- Removable implant supported overdenture

- Fixed implant prosthesis

Mandibular arch

- Extraction of remaining natural teeth and complete dentures
- Tooth supported overdenture

- Extraction of remaining natural teeth and implant supported overdenture

- Extraction of remaining natural teeth and fixed implant prosthesis

Taking the adequate interarch space, good periodontal prognosis of $33,34,43,44$, square-shaped mandibular arch, well-formed ridges, and financial status of the patient into consideration, it was planned to do a conventional complete denture for the edentulous maxillary arch and a tooth-supported overdenture with the Hader bar attachment on 33 and 43 and metal copings on 34 and 44 for the mandibular arch.

\section{Clinical Procedure}

- Intentional root canal treatment was carried out on the abutments $33,34,43$, and 44

- Tooth preparation was done with $33,34,43$, and 44 with a deep chamfer margin. Dome-shaped preparation was done for 34 and 44 (Fig. 3). Radicular preparation is done with 33 and 43 leaving 5-mm gutta-percha apically.

- Adequate tooth reduction was assessed using the previous maxillary denture made during the tentative jaw relation for adequate space for the arrangement of teeth and optimal crown to root ratio (Fig. 4).

- Chemicomechanical retraction was carried out using the 000 (Ultra pak, Prime Dental Pvt Ltd, India) retraction cord and 25\% of aluminum chloride (Hemostat Clear, Medikept UK Ltd, UK) 


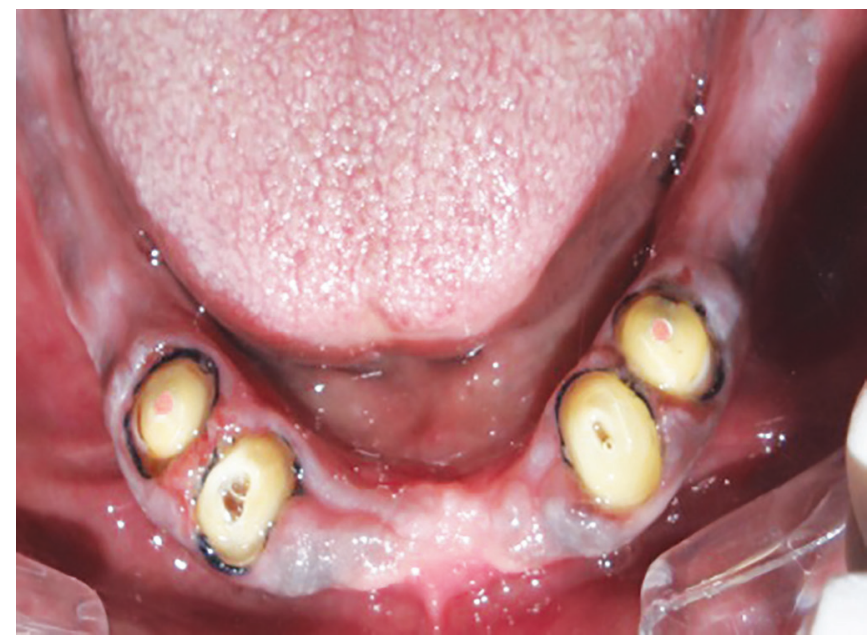

Fig. 3: Tooth preparation with 33, 34, 43, 44

and an impression was made using addition silicone putty and light body (Flexceed, GC India Pvt Ltd, India) (Fig. 5).

- The impressions were poured into a die stone (Ultra Rock, Kalabhai Karson Pvt Ltd., India). The distance between the two canine abutments was measured and a prefabricated bar of that length was used. The bar used was $4.5 \mathrm{~mm}$ in height (Fig. 6).

- Inlay wax pattern copings were made on $33,34,43$, and 44 and the prefabricated Hader bar was used to splint the copings on 33 and 43 (Fig. 7). This assembly was cast in the Co-Cr alloy. The castings were retrieved, finished, and polished following a standard procedure. A well-polished surface prevents the accumulation of plaque.

- The fitting of the bar attachment and the copings were assessed on the cast as well as intraorally (Fig. 8). A uniform space of $2 \mathrm{~mm}$ was present between the undersurface bar and the mucosa for the ease of cleaning.

- Custom trays were fabricated with a self-cure acrylic resin for the maxillary and the mandibular arch. Border molding with a low fusing impression compound followed by a wash impression with zinc oxide eugenol paste was carried out for the maxillary arch. Prior to the mandibular impression, the undersurface of the bar was blocked out using a boxing wax. Border molding

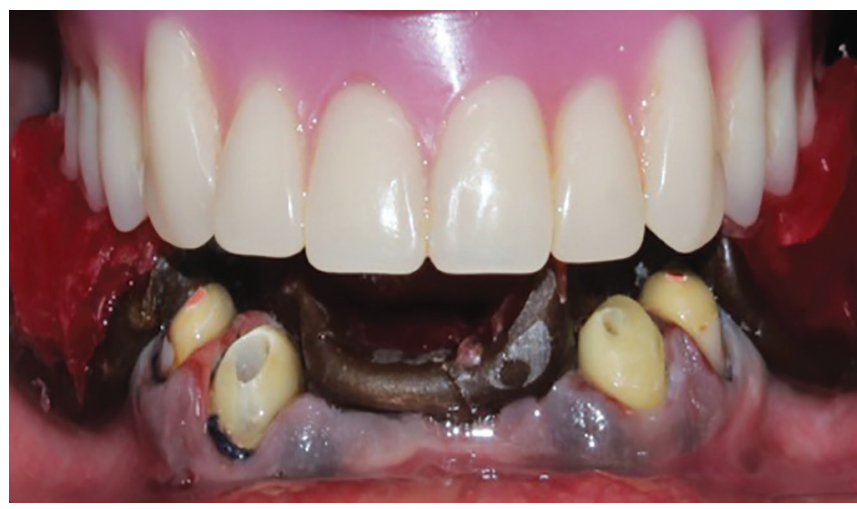

Fig. 4: Assessment of adequate reduction

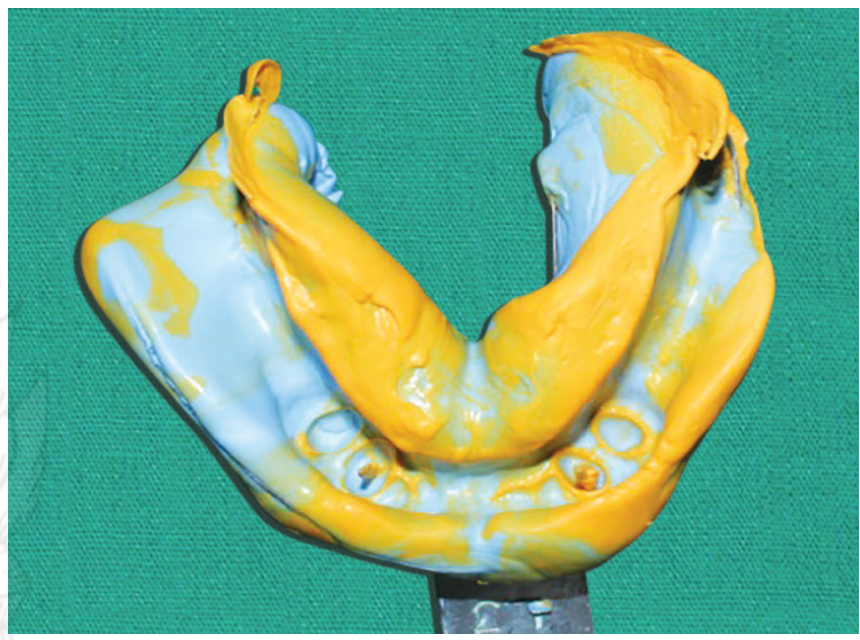

Fig. 5: Addition silicone impression

using a low fusing impression compound was carried out and a mandibular pick-up impression of the bar attachment and copings was made using light body addition silicone (Fig. 9).

- The master cast was poured into a die stone with the copings and bar attachment in position. The retentive clip and metal housing measuring $3 \mathrm{~mm}$ each were positioned on the bar and a duplicate impression was made (Fig. 10).
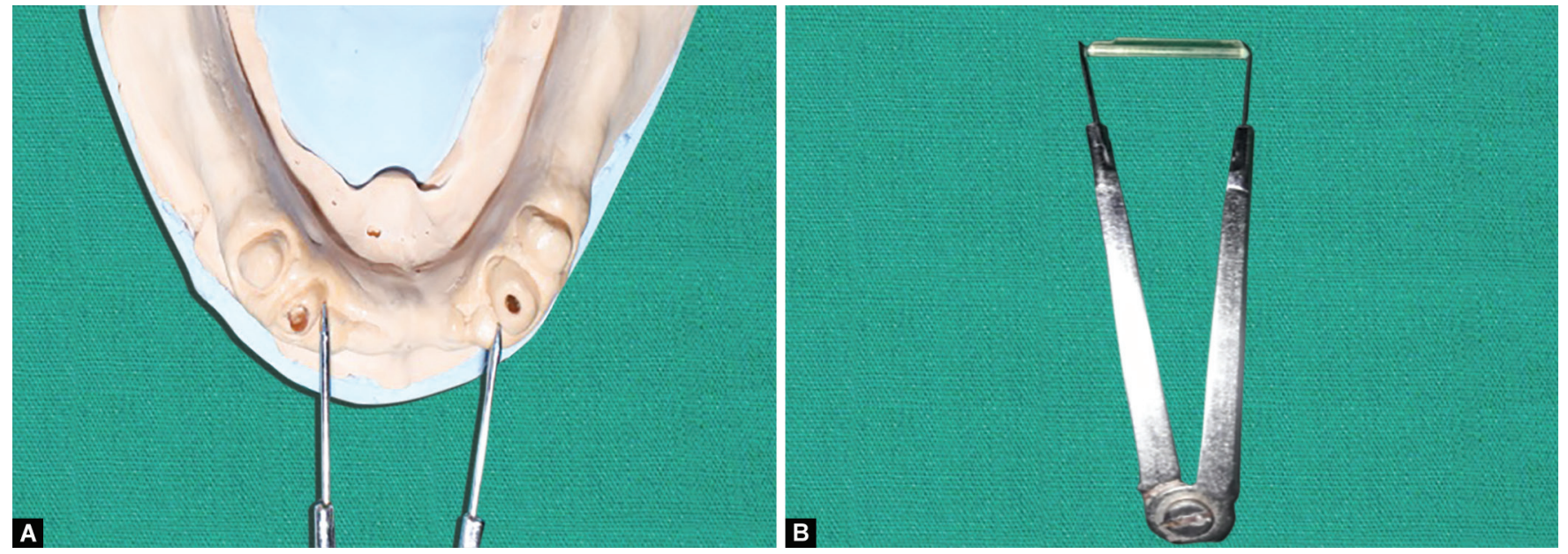

Figs $6 A$ and B: Measurement of length of the bar 


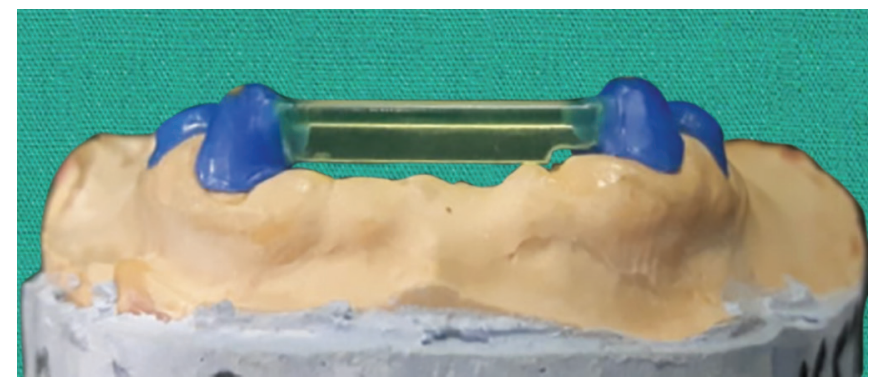

Fig. 7: Wax pattern of the bar on cast

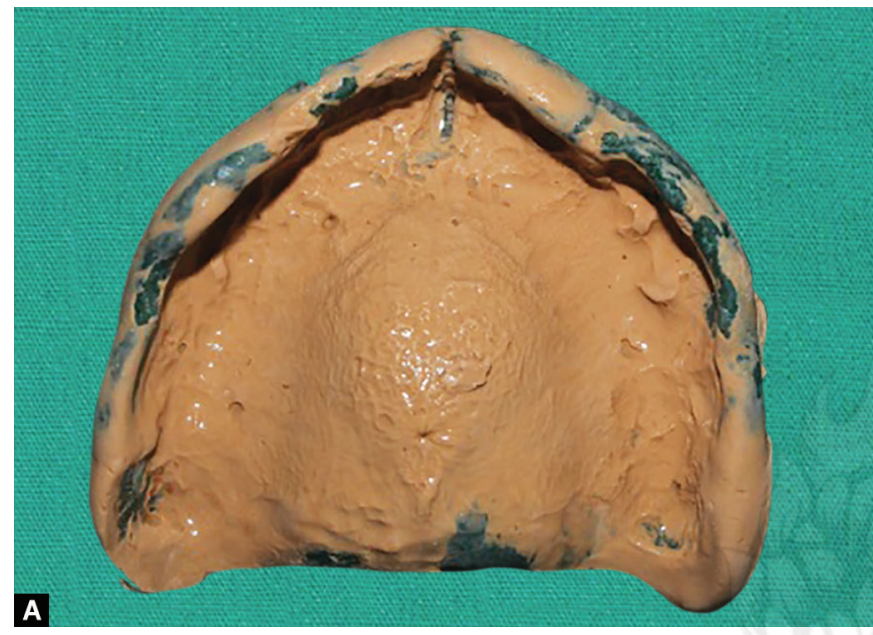

Figs $9 \mathrm{~A}$ and B: Maxillary and mandibular final impression

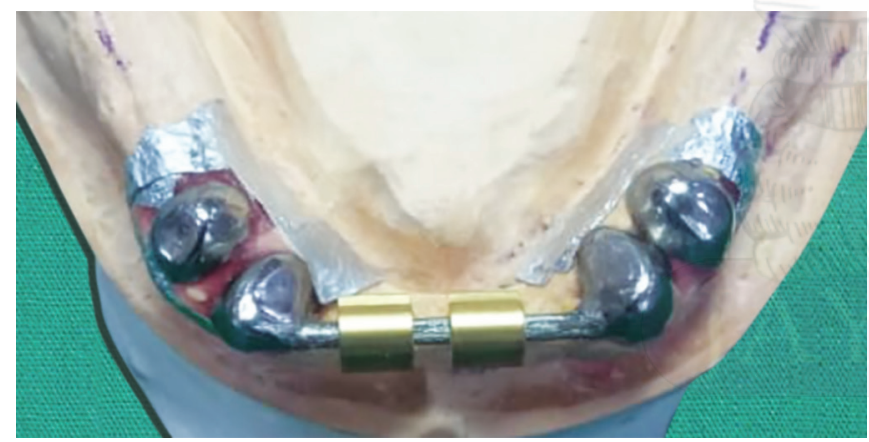

Fig. 10: Metal housing placed on the bar

- A metal mesh wax pattern was made on the refractory die and cast in the $\mathrm{Co}-\mathrm{Cr}$ alloy. This metal mesh acted by reinforcing the final acrylic denture to resist fracture (Fig. 11).

- A facebow record was made (Fig. 12). The jaw relation was recorded and transferred to the Hanau semiadjustable articulator. Teeth arrangement and trial of the denture were done to assess the fit and esthetics (Fig. 13).

- The bar attachment on the cast was blocked out and the plastic sleeve along with the metal housing was secured in the desired location. The acrylization process was carried out and the maxillary and mandibular dentures were retrieved, finished, and polished. The bar attachment along with metal copings was cemented using zinc phosphate cement.

- The mandibular denture was snapped into position with the aid of the retentive clips placed in the metal housings. The

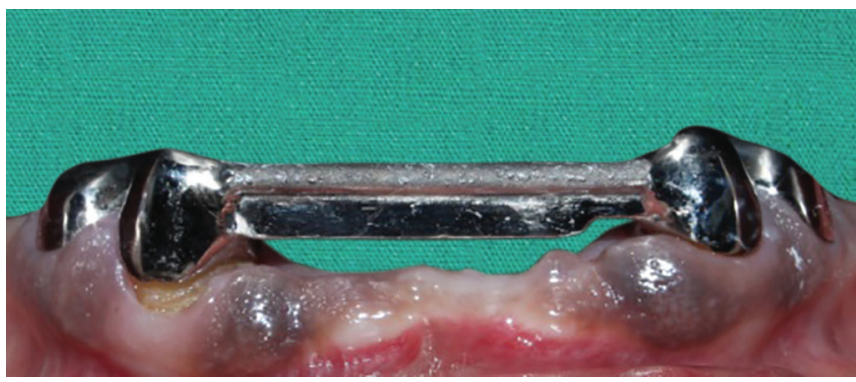

Fig. 8: Co-Cr bar intraorally
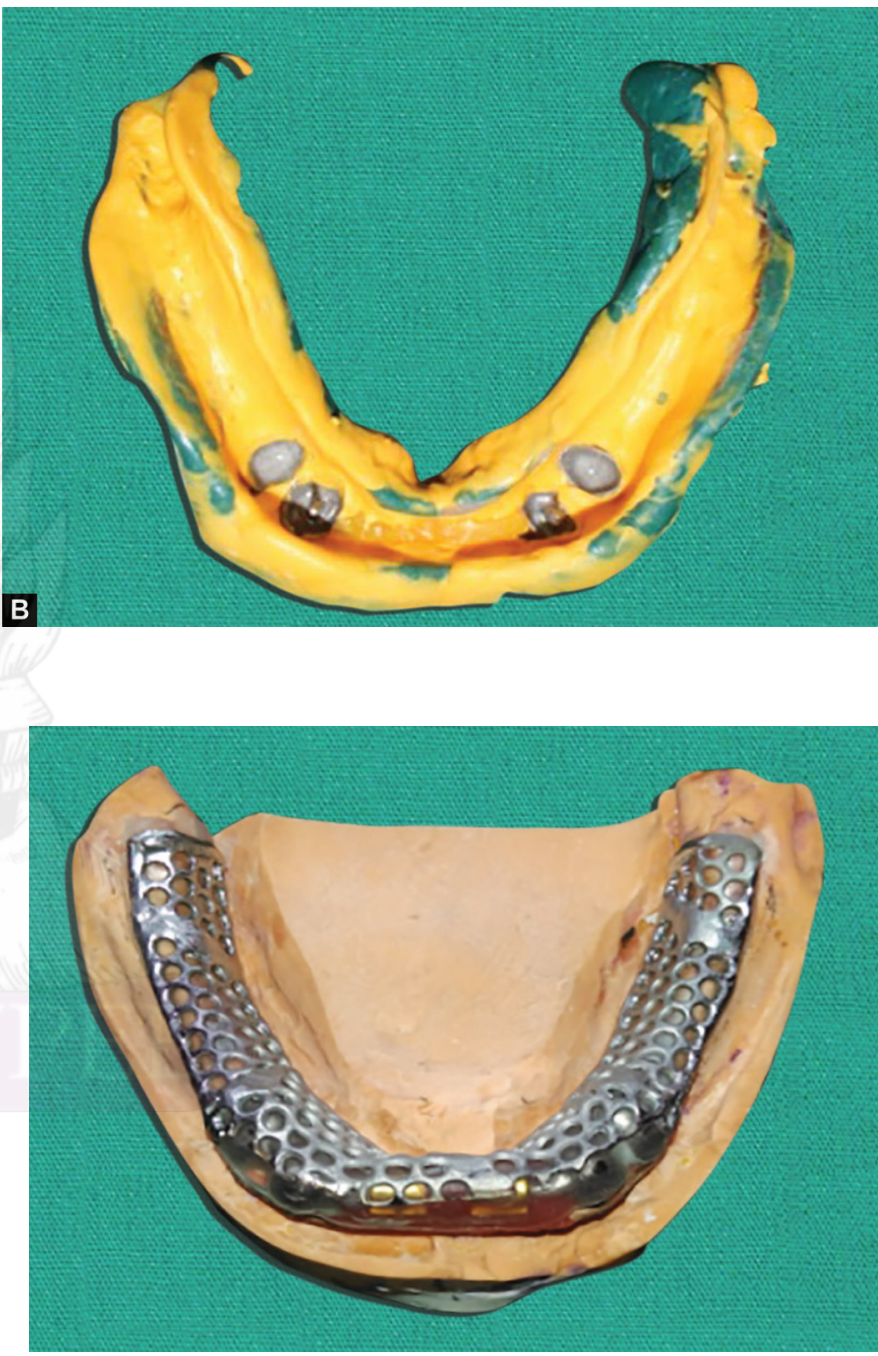

Fig. 11: Metal mesh

metal housing allows labiolingual movement of the clips during placement and removal of the prosthesis as well as secures the clip in position. The maxillary and mandibular dentures were assessed for retention, stability, support, comfort, and esthetics (Figs 14 to 16).

- The patient was requested to follow-up the next day and the following week for any adjustments. A 3-month follow-up was advocated.

- The patient was prescribed fluoride mouth wash and a Proxa brush to clean the undersurface of the bar. 


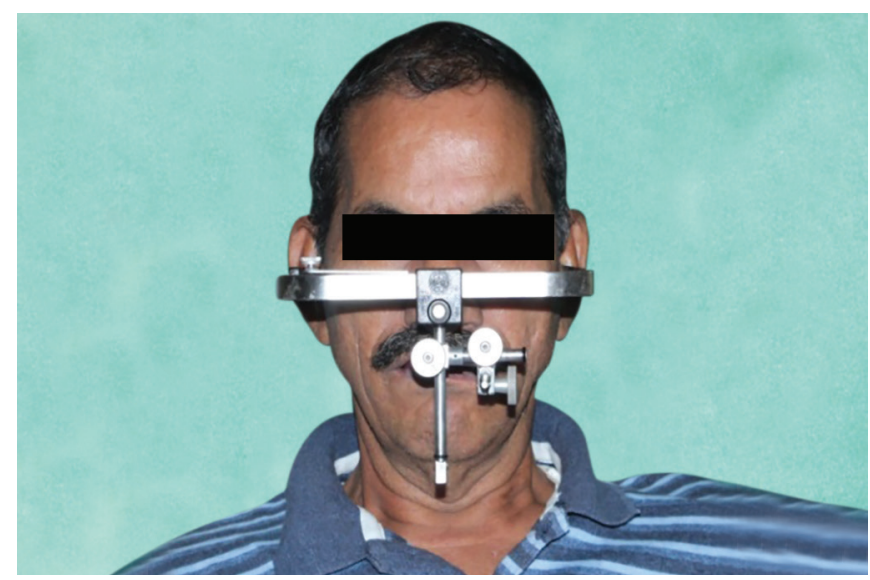

Fig. 12: Facebow record
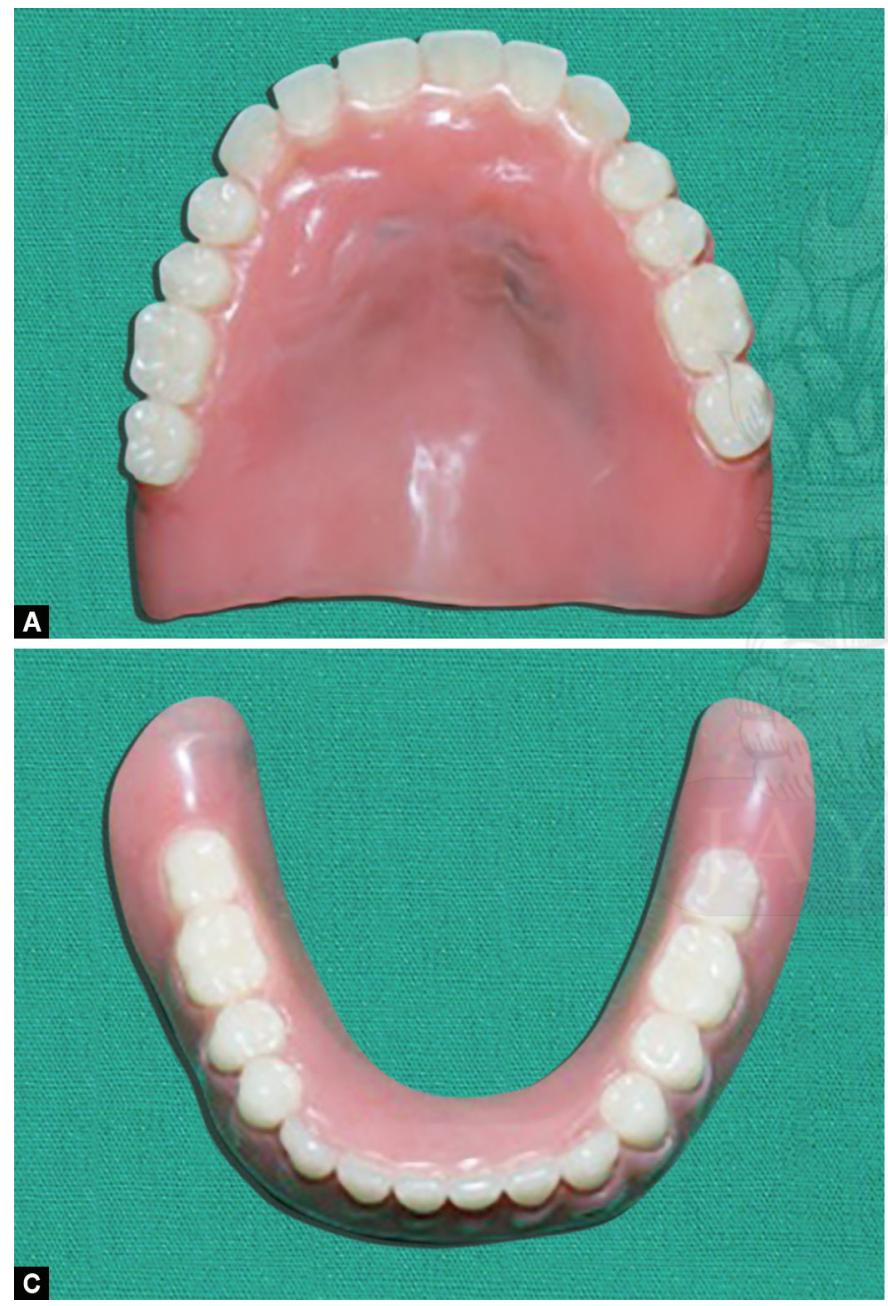

Figs 14A to D: Occlusal and polished surface of the denture

\section{Discussion}

Following the extraction of teeth, the maxillary and mandibular bones are subjected to life-long catabolic remodeling. The amount of bone loss to the resorptive process has been estimated at $21 \%$ by 3 months, 36\% after 6 months, and $44 \%$ after 1 year. ${ }^{8}$ Edward suggested that after the loss of natural teeth, the condyles, the

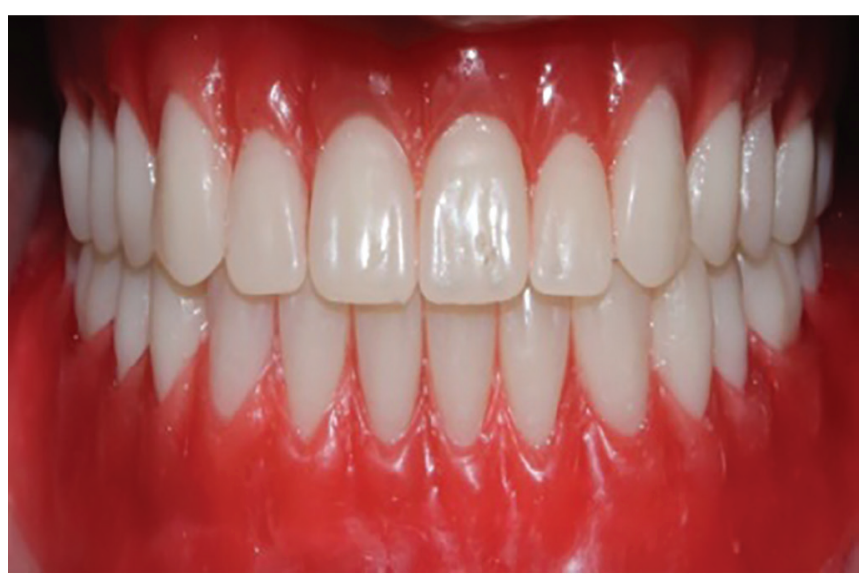

Fig. 13: Intraoral try-in
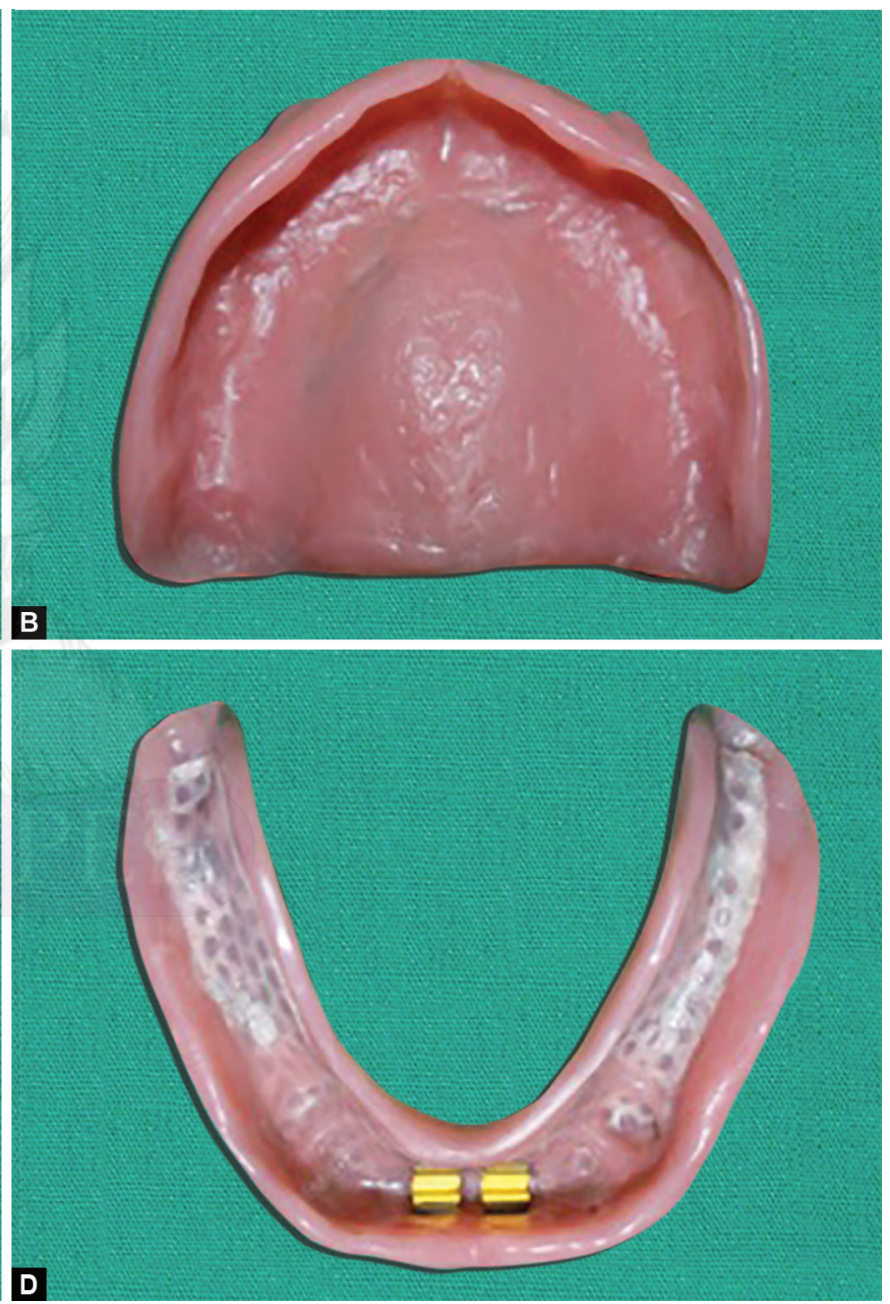

rami, and the body of mandible undergo changes due to long edentulous periods because of less intensive use of the masticatory muscles. ${ }^{9}$ It is a clinically proven fact that the bone loss is four times faster in the anterior mandible than in the anterior maxilla. Many denture wearers have difficulty in wearing and eating with mandibular complete dentures due to loss of retention and stability of the denture. The presence of the tongue moves the denture 


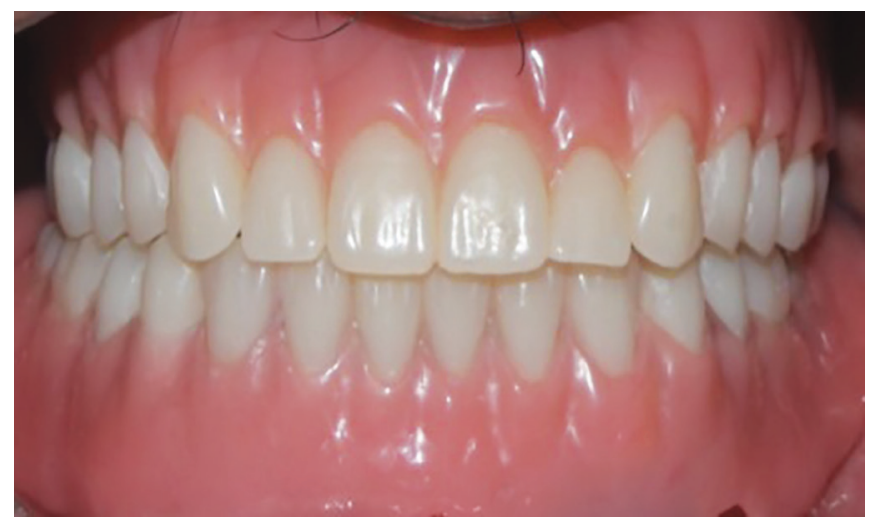

Fig. 15: Dentures in centric relation

while mastication. The obvious way to prevent this is by saving the natural teeth. The treatment option of saving the remaining natural teeth and constructing an overdenture has the advantages of enhanced stability retention and positive support of the denture as well as psychological benefit to the patient over the conventional complete denture. ${ }^{2,3}$ A study done by Rissin et al. concluded that the masticatory efficiency of overdentures was three times more than that of conventional complete dentures. ${ }^{10}$

Franco has classified overdenture as noncoping, with copings, and with attachments. Adequate interarch is a prerequisite in planning an overdenture with attachments. Overdentures with attachments distribute the occlusal forces evenly between the abutments and the remaining edentulous ridges thereby resulting in superior retention. ${ }^{11}$ Overdenture attachments are classified as extraradicular attachments and intraradicular attachments. In extraradicular attachments, studs which connect the prosthesis to individual tooth or bar attachments which splint the remaining abutments to distribute the forces.

Thayer and Caputo studied different bar designs and they concluded that the Hader bar distributed the occlusal forces more evenly between the posterior edentulous ridge and in between the abutments and produced less torquing forces than other bar designs. ${ }^{12}$ The Hader bar is a pear-shaped bar in cross section. The male portion is a metal bar which is attached to the abutment teeth by the means of copings with metal posts extending the intracanal root space splinting the abutment teeth and providing cross arch stabilization. The female component is a metal or plastic sleeve which is of the same diameter as the pear-shaped bar which is picked up in the impression surface of the denture. The presence of teeth maintains proprioception. ${ }^{13}$ The plastic sleeves or also called as the retentive clip positioned is placed in the metal housing flex during the placement of denture. The sleeve allows sagittal rotation and vertical translation from rest to function.

The use of the Hader bar attachments needs a square-shaped arch with strong abutments, adequate band of attached gingiva, and $15 \mathrm{~mm}$ or more interarch in each arch which was fulfilled in this case. Reducing the crown length reduces the crown to root ratio and, thus, reducing the mobility by $40 \%{ }^{13,14}$ The placement of the bar on the center of the ridge is essential. A more lingual position affects the movement of the tongue and a more buccal position hinders arrangement of teeth.

The clip insertion can be carried out directly in the patient mouth using autopolymerizing resin or indirectly using heat cured
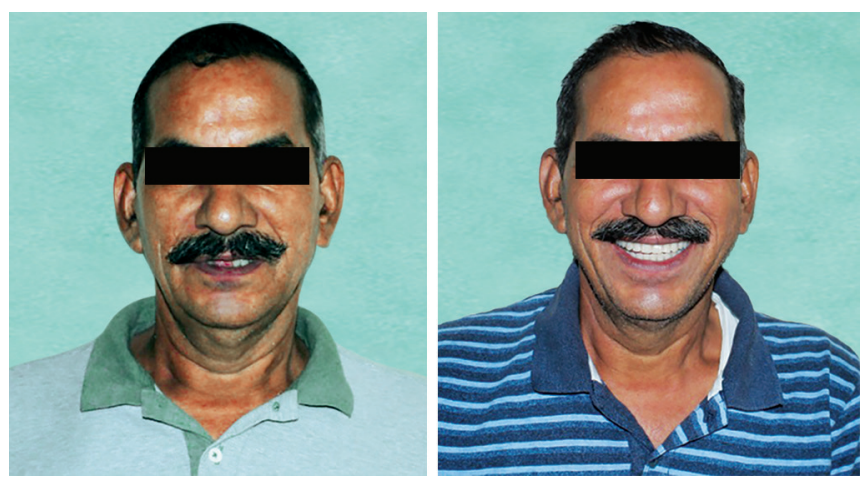

Fig. 16: Postoperative extraoral

acrylic resin via a laboratory procedure. In this case report, the indirect technique was advocated as it eliminates the disadvantages of autopolymerizing resin of water sorption, shrinkage, and more residual monomer affecting the clip insertion.

One of the major concerns for bar supported overdenture is meticulous oral hygiene is pertinent in order to prevent caries and periodontal diseases..$^{15,16}$ The bar in this study maintained no contact with the underlying tissue. Any contact between the bar and the mucosa causes compression leading to hyperplasia. The patient was educated to perform proper oral hygiene regimen including the use of proxy brush to clean the undersurface of the bar. ${ }^{17}$ Fracture of the denture is another concern which was taken care by incorporating metal mesh into the denture. Another disadvantage of using a bar attachment is the bulk of the denture, loss of retention of the retentive clip culminating in replacement of the clip.

\section{Conclusion}

Mandibular tooth or implant supported overdentures are a viable treatment option to preserve the remaining alveolar bone and maintain the proprioception, masticatory efficiency, and psychological benefit to the patient.

\section{Clinical Significance}

Various attachment systems are available and are considered a boon to prosthodontics with improved retention and stability of dentures. A detailed examination of the patient and proper selection of attachment according to the case dictates the success of tooth supported overdenture.

\section{References}

1. Samra RK, Bhide SV, et al. Tooth supported overdenture: a concept overshadowed but not yet forgotten! J Oral Res Rev 2015;7:16-21. DOI: $10.4103 / 2249-4987.160172$

2. Morrow RM, Feldmann EE, et al. Tooth supported complete dentures. An approach to preventive prosthodontics. J Prosthet Dent 1969;21:513-522. DOI: 10.1016/0022-3913(69)90073-0.

3. Dodge CA. Prevention of complete denture problems by use of "Overdenture". J Prosthet Dent 1973;30:403-406. DOI: 10.1016/00223913(73)90161-3.

4. The glossary of prosthodontic terms (GPT 9); 2017. p. e65.

5. Bascom PW. Preservation in Prosthodontics. J Prosthet Dent 1971;25:489-492. DOI: 10.1016/0022-3913(71)90204-6. 
6. Crum RJ, Loiselle RJ. Oral Perception and Proprioception: A Review of the Literature and Its Significance to Prosthodontics. J Prosthet Dent 1972;28:215-230. DOI: 10.1016/0022-3913(72)90141-2.

7. Schwartz IS, Morrow RM. Overdentures. Principles and procedures. Dent Clin North Am 1996;40:169-194.

8. Carlsson G, Persson G. Morphologic changes of mandible after extraction and wearing of dentures. Odontol Rev 1967;18:27-32.

9. Edwards LF. The Edentulous Mandible. J Prosth Dent 1954;4:222. DOI: 10.1016/0022-3913(54)90100-6.

10. Rissin L, House JE, et al. Clinical comparison of masticatory performance and electromyographic activity of patients with complete dentures, overdentures, and natural teeth. J Prosthet Dent 1978;39(5):508-511. DOI: 10.1016/S0022-3913(78)80181-4.

11. Thayer HH. Overdentures and the periodontium. Dent Clin North Am 1980;24:369-77.
12. Dolder EJ. The bar joint mandibular denture. J Prosthet Dent 1961;11(4):689-707. DOI: 10.1016/0022-3913(61)90178-0.

13. Thayer HH, Caputo AA. Photoelastic stress analysis of overdenture attachments. J Prosthet Dent 1980;43:611-617. DOI: 10.1016/00223913(80)90374-1.

14. Dong J, Ikebe K, et al. Influence of abutment height on strain in a mandibular overdenture. J Oral Rehabil 2006;33:594-599. DOI: 10.1111/j.1365-2842.2005.01602.x.

15. Brill N, Schfibeler $\mathrm{S}$, et al. Aspect of occlusal sense in natural and artificial teeth. J Prosthet Dent 1962;12:123.

16. Manly RS, Braley LC. Mastlcatory performance and efficiency. J Dent Res 1950;29:448-462. DOI: 10.1177/00220345500290040701.

17. Kapur KK, Soman S. Masticatory performance and efficiency in denture wearers. J Prosthet Dent 1964;14(4):687-694. DOI: 10.1016/0022-3913(64)90203-3. 